\title{
Differential Regulation of Functional Gene Clusters in Overt Coronary Artery Disease in a Transgenic Atherosclerosis-hypertensive Rat Rodel
}

\author{
Victoria L. M. Herrera, Tamara Didishvili, Lyle V. Lopez, and Nelson Ruiz-Opazo \\ Unit of Molecular Genetics, Whitaker Cardiovascular Institute, Section of Molecular Medicine, Evans \\ Department of Medicine, Boston University School of Medicine, Boston, MA, USA \\ Accepted June 10, 2002
}

\begin{abstract}
Background: Human acute coronary syndrome refers to the spectrum of clinical manifestations of overt coronary artery (CAD) disease characterized by atherosclerotic plaque destabilization and resultant myocardial injury. Typically studied as distinct pathologies, emerging pathogenic paradigms implicate multiple processes beyond thrombosis and ischemic cell injury respectively, with significant pathway overlap involving inflammation, apoptosis, matrix degradation, and oxidative stress. However, all these pathways have also been implicated in stillquiescent coronary plaque progression, thus making it harder to pinpoint the turnkey events leading to overt-CAD. Analysis of transcription profiles could identify a working framework of pathogenesis distinguishing overt-CAD.

Materials and Methods: We investigated the transcription profile associated with overt-coronary artery disease (CAD), in contrast to quiescent-CAD and attenuated, quiescent-CAD using the Tg53 transgenic atherosclerosishypertensive rat model, which exhibits end-stage coronary heart disease simulating human acute coronary syndromes. Using a rat-specific known-gene oligonucleotide array, twice corroborated transcription profiles from four individual Tg53 rats exhibiting overt-CAD were
\end{abstract}

analyzed and contrasted to transcription profiles of agematched Tg53 rats with quiescent-CAD (pooled $\mathrm{n}=4$ ) and attenuated, quiescent-CAD (pooled $n=4)$.

Results: $\operatorname{Tg} 53$ male rats with overt-CAD exhibited distinct transcription profiles compared with both quiescent-CAD control groups. Functional gene cluster analysis detects upregulation of genes involved in inflammation (interleukin-1, interleukin-18, Fc $\gamma$ II receptor, thyrotropin releasing hormone), matrix balance (membrane type metalloproteinase, TIMP-1, lysyl oxidase), oxidized LDL entry (endothelial oxLDL receptor), which contrast deinduced gene clusters involved in angiogenesis, proliferation, metabolism, ion transport and adrenergic receptors. Conclusions: The data demonstrate that transcriptionally mediated events distinguish the onset of overt-CAD and identify a first list of putative "turnkey" genes. This altered molecular framework implies an altered "hardwiring" which a priori would require multifaceted, targeted interventioncurrently not implemented to date. Although more studies are necessary, early concordance with current pathogenic paradigms of human coronary plaque destabilization and post-ischemic myocardial response provides translational significance to observations and hypotheses.

\section{Introduction}

Human coronary atherosclerosis is a multifactorial disease, which develops insidiously over decades progressing from quiescent to overt life-threatening coronary artery disease (CAD) clinically known as acute coronary syndromes-the spectrum of unstable angina, acute myocardial infarction and sudden coronary death $(1,2)$. Pathogenically, acute coronary syndrome is the clinical manifestation of two concurrent pathological processes: a vascular pathology marked by "culprit" atherosclerotic plaques and myocardial pathology marked by ischemia and/or infarction (3-5), hence typically studied separately. Comparative analysis of emerging paradigms from these studies highlights parallel cellular events involving inflammation, apoptosis, matrix degradation, neutrophil recruitment in both pathogenic

Address correspondence and reprint requests to: Victoria L. M. Herrera, Whitaker Cardiovascular Institute-W609, 700 Albany Street, Boston, MA. 02118, USA, Phone: (617) 638-4020, fax: (617) 638-4066, e-mail: vherrera@bu.edu. hallmarks of overt-CAD-the destabilized "culprit" plaque (3) and post-infarction myocardium (4). These parallel changes reflect either an interesting, albeit inconsequential coincidence or a putative central interactive paradigm. The challenge therefore lies in the elucidation of the pathogenic framework involved in overt-CAD as a first step, and the subsequent delineation of mechanisms within and cross talk between vascular and myocardial pathological events. The notion of cross-talk seems intuitively pertinent given that circulating elevated inflammatory cytokines would affect both vascular and myocardial events. Critical fist-step analysis in validated animal models of coronary artery disease becomes key.

Transcription profiling offers an investigative paradigm that can identify framework concepts of pathogenesis and specific gene pathways once confounding variables are regulated. The existence of confounding variables, however is inherent in acute coronary syndromes given the characteristic heterogeneity of "culprit plaques" (5) and differential 
genetic susceptibility to atherosclerotic plaque formation (6). These features underscore an inherent complexity in the progression from quiescent- to overt-CAD and reiterate the need for first-line transcription profiling investigation in validated surrogate experimental systems, wherein confounders can be experimentally controlled while maintaining potential for integrated analysis. A priori, the presence of a reproducible transcriptional profile that distinguishes overt-CAD from quiescent-CAD would imply that an active transcriptional process is central to the pathogenesis of overt-CAD. Determining whether there is indeed a differential transcription profile in overt-CAD would provide critical insight into detection, intervention and prevention pathways.

In order to eliminate confounding variability from differential genetic susceptibility, age, diet, activity and tissue-source variability, we investigated the transcription profile of cardiac right ventricle from transgenic Tg53 rat model of coronary artery disease $(7,8)$ at the onset of cardio-respiratory distress-taken as the experimental equivalent of acute coronary syndromes. Although limited to 1046-known-rat gene array, our results reveal reproducible, differential functional gene-cluster regulation with putative circuitous links among key genes. These observations differ from the transcription profile reported in coronary-ligation ischemia mouse model (9), thus forwarding the hypothesis that distinct transcriptional mechanisms are central to the pathogenesis of overt-CAD.

\section{Materials and Methods}

\section{Experimental Design and Tissue Source}

Rat experiments were conducted according to Institutional Animal Care and Use Committee guidelines. Identical genetic background of the $\mathrm{Tg} 53$ rats in the inbred Dahl salt-sensitive hypertensive rat strain eliminates variability from genetic modifiers and/or susceptibility to lesion formation and vascular phenotypes (7). Environmental factors were regulated through identical housing, manipulation, and pathogen-free maintenance, thus eliminating stress and infection as potential confounding variables. All animals are gender- and age-matched in order to eliminate these clinically significant variables as confounders. Diet was defined as to study groups so that we could compare overt-CAD with quiescent-CAD rats on regular salt $(\approx 0.4 \% \mathrm{NaCl}$, PicoLab Rodent Diet 20, PicoLab, Richmond, IN) diet $\left(\mathrm{q}-\mathrm{CAD}^{\mathrm{R}}\right)$, as well as attenuated quiescent-CAD rats on low salt $(0.004 \% \mathrm{NaCl}$, Harlan Teklad, Inc., Madison, WI) diet $\left(\mathrm{aq}-\mathrm{CAD}{ }^{\mathrm{L}}\right)$. Right ventricles were collected from Tg53 rats exhibiting overt-CAD, quiescent-CAD ${ }^{\mathrm{R}}$ and quiescent-CAD ${ }^{\mathrm{L}}$-all at 8 months of age. Total cellular RNAs were isolated using Triazol reagents as per manufacturer's specifications (Gibco-BRL, Rockville, MD, USA). RNA sample purity, non-degradation and quantity were verified by paraformaldehyde-denaturing agarose gel electrophoresis prior transcription profiling analysis.

\section{Transcription Profiling and Analysis}

Transcription profiling was done using the rat-specific oligonucleotide-based array containing 1046 unique genes and 109 additional gene spots for negative, positive and quantitative controls based on 3' untranslated gene sequences thus ascertaining transcript specificity (Mergen Ltd., CA, USA). Expression levels were analyzed using ImaGene4.0 BioDiscovery data analysis software (Mergen Ltd., CA, USA). Confidence with array expression profiles was obtained by concordance with known cardiac gene expression derived from previous RNA blot analysis. Transcription profile expression changes noted for overt CAD were limited to changes in Tg53[o-CAD $\left.{ }^{\mathrm{R}}\right]$ confirmed twice in two independent array analysis, as well as concordance in 4 individual $\mathrm{Tg} 53\left[\mathrm{o}-\mathrm{CAD}^{\mathrm{R}}\right]$ samples. Expression changes were taken only from those comparison-pairs wherein at least one or both samples are above threshold after subtracting background. Two-fold changes and greater were noted, as well as 1.5 to 1.7 -fold change because of $2 \mathrm{x}-$ corroboration per overt-CAD sample $(n=4)$, as well as concordance with both quiescent-CAD $\left(\mathrm{qCAD}^{\mathrm{R}}\right.$ and $\mathrm{qCAD}^{\mathrm{L}}$ ) control samples which represented a pool of 4 rats for group accuracy. Transcription levels were normalized by subtracting local background levels; threshold is defined as local background. Values greater than threshold are normalized values greater than background.

Analysis was done with Excel-based sequential sorting. Reproducibility and levels greater than detection threshold values were ascertained. Genes were then assigned to functional gene clusters based on published reports. Interactions were assigned based on reported functional interactions by different research groups. Pattern analysis was also done on rank order of expression levels with the following sequence: o-CAD, q-CAD, and aq-CAD. A gradientpattern of expression levels (graded as $1^{\mathrm{o}-\mathrm{CAD}}>$ $2^{\mathrm{q}-\mathrm{CAD}}>3^{\mathrm{aq}-\mathrm{CAD}}$ or $3>2>1$ ) was deduced to most likely reflect lesion progression. Likewise, assignment to lesion and/or cardiac changes was deduced if pattern of expression levels exhibited change only in overt-CAD (graded as $1^{\mathrm{O}-\mathrm{CAD}}, 3,3$ or $3^{\mathrm{O}-\mathrm{CAD}}, 1,1$ ).

\section{Immunohistochemical Analysis}

TIMP-1 immunohistochemical analysis was done essentially as described on serial sections of culprit plaques identified previously (8). TIMP-1 antibody from Sta. Cruz Biotechnology (CA, USA) was used.

\section{Results}

We investigated the transcription profile of right ventricles with different stages of CAD in the inbred transgenic hyperlipidemic-polygenic hypertension 
Tg53 rat model (7). This model develops significant coronary atherosclerosis simulating key histological features of "culprit" coronary plaques in human acute coronary syndromes (8). We compared 8 montholdTg53 male rats with clinically overt-CAD and quiescent-CAD with equivalent hyperlipidemia and hypertension on regular rat chow $\left(\mathrm{qCAD}^{\mathrm{R}}\right)$, as well as with age-matched rats with attenuated, quiescent-CAD on low salt diets $\left(\mathrm{aqCAD}^{\mathrm{L}}\right)$ resulting in equivalent hyperlipidemia but decreased hypertension (8). We selected the right ventricle (RV) as tissue source for transcription profiling since coronary atherosclerosis in this model is more robust in the RV than the left ventricle (LV), and being thinwalled and non-hypertrophied compared to the LV, it provides a relative experimental enrichment of coronary artery disease pathology, thus allowing the integrated analysis of both vascular and myocardial events in overt-CAD. Parameters for a stringent experimental design were followed to ascertain robustness of transcription profiling results. Transcription profiling was done using a rat-specific array containing 1046 unique genes and 109 additional gene spots for negative, positive and quantitative controls.

Scatter plots of gene array data from right ventricular RNA samples provide an overview of comparative transcription profiles (Fig. 1). Most genes are expressed above the array technical threshold-a stringent parameter designed to eliminate false positives. Of 1046 unique genes on the array used, only a minority exhibit $>2$-fold vectorial change marked as outliers above or below the redlined 2-fold cutoff (Fig. 1). Differential analysis of transcription profiles of pooled $(n=4)$ right ventricular total cellular RNA from Tg53 male rats on regular rat chow exhibiting overt $\mathrm{CAD}[\mathrm{o}-\mathrm{CAD}]^{\mathrm{R}}$ highlights 2 -fold vectorial changes in gene expression compared with pooled $(n=4)$ right ventricular total cellular RNA from control age- and diet-matched $\operatorname{Tg} 53$ male rats with quiescent, empirically asymptomatic CAD $[\mathrm{q}-\mathrm{CAD}]^{\mathrm{R}}$ (Fig. 1), as well as when compared with Tg53 rats with attenuated, quiescent-CAD on low salt diet $(\mathrm{n}=4)(8), \operatorname{Tg} 53[\mathrm{aq}-\mathrm{CAD}]^{\mathrm{L}}$ (Fig. 1B). As would be expected from an attenuated phenotype on low salt diet (8), more genes exhibit $>2$-fold induction when comparing overt-CAD in Tg53[o-CAD $]^{\mathrm{R}}$ rats with attenuated, quiescent-CAD $\operatorname{Tg} 53$ [aq-CAD] ${ }^{\mathrm{L}}$ rats (Fig. 1B), in contrast to the transcription profile comparison between overt- and quiescent (nonattenuated) CAD (Fig. 1A). Transcription profile comparison of both quiescent CAD controls, Tg53 $[\mathrm{q}-\mathrm{CAD}]^{\mathrm{R}}$ vs. $\operatorname{Tg} 53[\mathrm{aq}-\mathrm{CAD}]^{\mathrm{L}}$, reveals quite similar expression profiles (Fig. 1C), thus providing confidence in the detected differential transcription profile in overt-CAD compared with two different control groups. A priori, a differential transcription profile implies differential molecular framework in overt-CAD.

In order to gain insight into said differential gene pathways, we then dissected the differential expression profiles revealed in Figure 1 for functional gene clusters. First, we filtered for consistency of vectorial individual gene expression changes detected in overt CAD compared to both quiescent CAD controls. We defined consistency of gene expression changes as o-CAD $>\mathrm{q}-\mathrm{CAD}^{\mathrm{R}} \geq \mathrm{qa}-\mathrm{CAD}^{\mathrm{L}}$, or inversely qa-CAD ${ }^{\mathrm{L}} \geq \mathrm{q}-\mathrm{CAD}{ }^{\mathrm{R}}>\mathrm{o}-\mathrm{CAD}$. Second, we filtered for reproducibility in independent array experiments. Only changes that were detected reproducibly when RV samples were analyzed individually
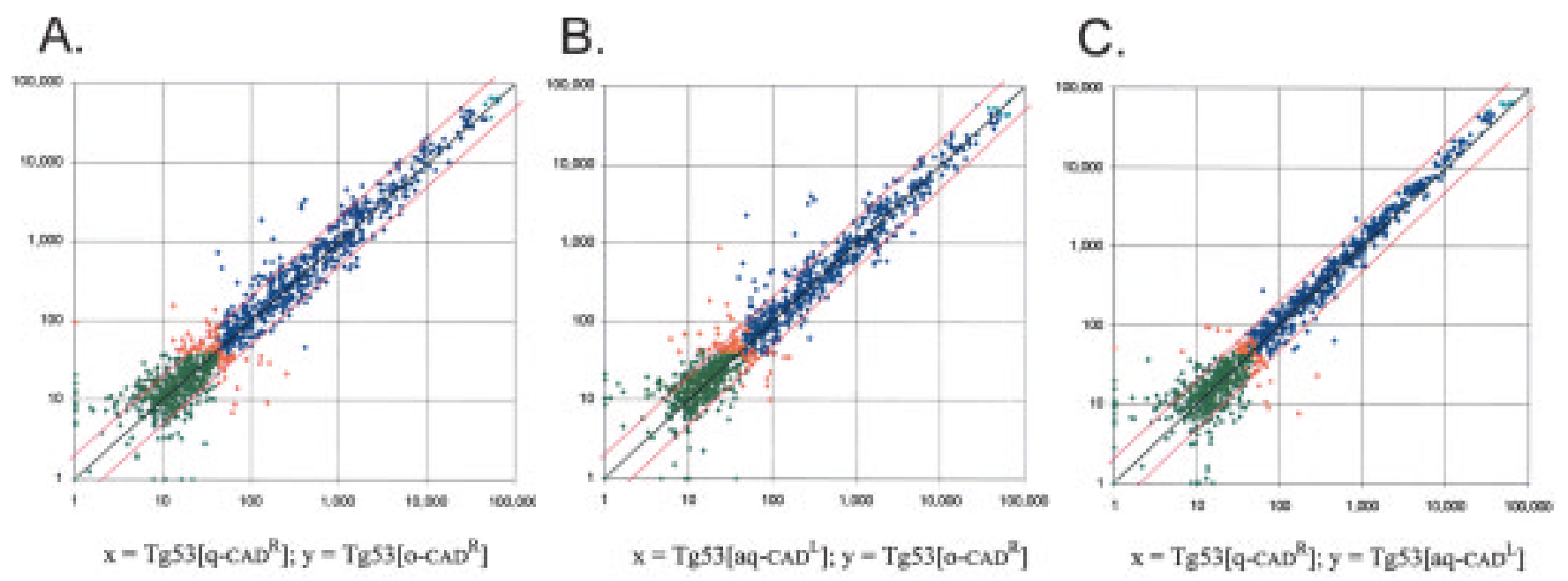

Fig. 1. Overview of transcription profile changes in overt-CAD vs quiescent-CAD. Scatter plots of right ventricular transcription profiles reveals genes with significant number of genes with $>2$-fold expression changes between $(A)$, quiescent $C A D\left[q-C A D^{R}\right]$ and overt-CAD [o-CAD $\left.{ }^{\mathrm{R}}\right] ;(\mathrm{B})$, attenuated quiescent-CAD [aq-CAD $\left.{ }^{\mathrm{L}}\right]$ and overt-CAD $\left[\mathrm{o}-\mathrm{CAD}{ }^{\mathrm{R}}\right] .(\mathrm{C})$, Scatter plot comparing quiescent $\mathrm{CAD}\left[\mathrm{q}-\mathrm{CAD}{ }^{\mathrm{R}}\right]$ and attenuated $\mathrm{CAD}\left[\mathrm{aq}-\mathrm{CAD}^{\mathrm{L}}\right]$ reveals minimal $>2$-fold gene expression changes., green $=$ expression level below array threshold for both; $\odot$, orange = expression level of one sample below threshold; $\bullet$, blue = expression levels of both sample above threshold; , aqua $>$ maximum intensity. Units $1-10,000=\log 10$ (signal-local background) intensity of fluorescence signal. 
and when pooled $(n=4)$ are presented. Third, we filtered for robust detection signals. Gene expression changes are noted only if the normalized expression level (signal-local background) is greater than threshold (local background) in the overt CAD samples or in both quiescent-CAD control groups. Genes with consistent $\geq 1.5$-fold vectorial change in expression in overt-CAD compared with both control groups for quiescent-CAD are delineated. Although the typical $\geq 2$-fold change has to date become the emerging standard for accepting vectorial fold-changes in array analysis to minimize spurious results, we report $\geq 1$.5-fold changes that have exhibited reproducibility in independent experiments thus eliminating risk of spurious results. Assignments were then done based on the pattern of gene expression levels-gradient pattern implying worsening plaque changes; step-change pattern implying association with cardiac compromise and/or plaque compromise. After this quantitative filtering, qualitative functional gene clustering was done based on known gene function or when unknown, based on homology with known genes.

Of the 1046 unique genes on the rat array used, $568(54 \%)$ are expressed above array threshold in 8 month-old $\operatorname{Tg} 53$ rats with overt-CAD. Of these, only $2.3 \%$ are induced and $3.7 \%$ are deinduced in overt-CAD compared with age-matched $\mathrm{Tg} 53$ rats with quiescent-CAD. Assuming randomness of genes on the array, gene expression changes stratify into distinct functional gene clusters between induced versus de-induced genes (Fig. 2). Interestingly, $69 \%$ of induced genes belong to functional gene clusters implicated in coronary plaque destabilization such as matrix regulation 4/13, inflammation $4 / 13$, and oxidized LDL entry $1 / 13$. These functional gene groups are distinct from deinduced gene clusters (Fig. 2), which involve energy metabolism 9/21, proliferation 5/21, $\alpha$-adrenergic system $3 / 21$, and angiogenesis $2 / 21$.

In addition to stringent parameters of consistency and robustness of expression signals among study individual and pooled samples, within-array reproducibility of gene expression levels was also observed for both positive and negative controls, and more importantly for markedly increased genes such as TIMP1 and membrane-type matrix metalloproteinase (Fig. 2). Moreover, immunohistochemistry with TIMP-1 antibody detects expression in macrophage-foam cells of proximal high-risk lesions rather than in the myocardiocytes or more distal coronary stable lesions (Fig. 3, A-D). This provides confidence in the significance of array-based observations of expression levels. Interestingly, immunohistochemical analysis detects a deinduction of TIMP-1 expression in endothelial cells overlying lesions in overt-CAD (Fig. 3, A-C) compared with attenuated quiescent-CAD ${ }^{\mathrm{L}}$ (Fig. 3, E-F). The deinduction is specific to endothelial cells overlying "culprit" lesions-and contrasts the strong expression in endothelial cells of microvasculature in the adjacent myocardium (Fig. 3, A-C), which provide an experimental positive control on the same section.

Analysis of the functional clusters exhibiting differential gene changes identifies putative gene networks involved in overt-CAD. Based on concordance with published research, gene pathways can be assigned to either or both hallmark pathologies of overt-CAD-plaque destabilization and myocardial destabilization post-ischemia/infarction (Fig. 4). Confidence in these publication-based assignments can be derived from concordance with lesion or \{lesion + cardiac\} assignments predicted by rank order of expression levels among the three study groups (Fig. 2). Assignment to lesion-based expression pattern is likely when the rank order reflects a gradient-either $3^{\mathrm{o}-\mathrm{CAD}}>2^{\mathrm{q}-\mathrm{CAD}}>1^{\mathrm{aq}-\mathrm{CAD}}$ or $1^{\mathrm{O}-}$ CAD $<2^{\mathrm{q}-\mathrm{CAD}}<3^{\mathrm{aq}-\mathrm{CAD}}$-since the variable among the three study groups that is consistent with a gradient is severity of lesion pathology. When the rank order reflects a step-change in overt-CAD-either $3^{\mathrm{O}-\mathrm{CAD}}>1=1$ or $1^{\mathrm{O}-\mathrm{CAD}}<3=3$, distinction cannot be made hence assignment to \{lesion + cardiac\}-based expression pattern. Although these assignments need to be experimentally confirmed by cell-specific analysis, they provide supportive concordance to functional cluster analysis of putative pathways.

More specifically, pathway analysis highlights several putative paradigms (Figs. 2, 4). First, a paradigm reminiscent of tumor invasiveness brings coherence to matrix degradation, inflammation, and apoptosis-with induction of MT-MMP, lysyl oxidase, TIMP-1, IL-1 beta, IL-18-all genes reported to be associated respectively with tumor invasiveness (10-15). A tumor-invasiveness paradigm would be consistent with both hallmark pathologies of overtCAD-plaque destabilization and ensuing myocardial destabilization post-ischemia/infarction. Secondly, a paradigm of decreased proliferation-with decrease of growth factors, transcription factors and cell cycle regulators, as well as increase of a suppressor of proliferation, TSC-22-suggests a pathway underlying the histological endpoint of fibrous cap weakness in high-risk plaques marked by decreased number of smooth muscle cells and fibroblasts. Since decreased proliferation is unlikely to be a cardiomyocyte event, and given that with the exception of one, genes involved with decreased proliferation are assigned to lesion expression pattern by rank order (Fig. 2), decreased proliferation pathways can be hypothesized to be part of a lesion destabilization paradigm. Experimental validation remains to be done. Thirdly, a paradigm of lipid-associated and lipid-immune complex triggers-with respective increased oxLDL endothelial receptor and macrophage/neutrophil Fc $\gamma$ II receptorprovides a plausible initiator of inflammatory cascade(s) leading to lesion destabilization and/or coronary dysfunction. A fourth paradigm represents changes in functional gene clusters that reflect an altered myocardial functional state: $\alpha \mathrm{l}$ adrenergic 


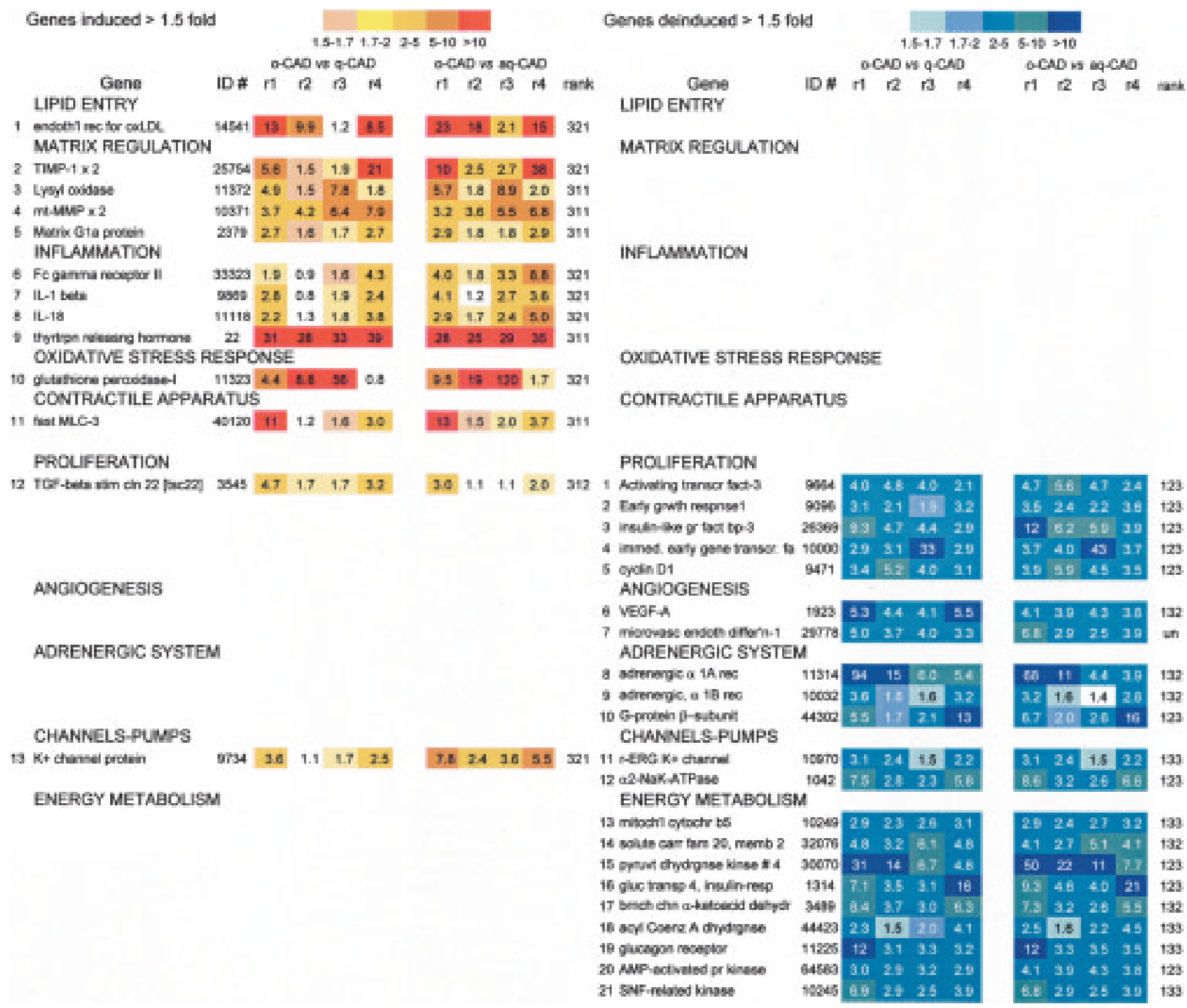

Fig. 2. Functional gene-cluster specific differential regulation-turnkey genes. Based on known gene function, genes that exhibit consistent vectorial change ( $>1.5$ fold) in gene expression in overt-CAD compared with both quiescent-CAD control groups fall into the following functional clusters: lipid entry, matrix balance, inflammation, oxidative stress response, contractile apparatus, proliferation, angiogenesis, adrenergic system, channels-pumps, energy/metabolism. Induction of gene expression detected spans 1.5 to 40 -fold; deinduction spans 1.5 to 40 -fold; fold expression $<1.5$ not color coded. Induced functional gene clusters are distinct from deinduced functional gene clusters. Pattern of expression changes were identified by ranking levels of expression in the following sequence: overt o-CAD, quiescent $\mathrm{q}-\mathrm{CAD}$ and attenuated, quiescent aq-CAD as noted: gradient pattern (rank $32 \mathrm{l}$ : $3^{\mathrm{O}-\mathrm{CAD}}>2>1$ or rank 123: $1^{\mathrm{O}-\mathrm{CAD}}<2<3$ ); step-change pattern (rank 133: $1^{\mathrm{O}-\mathrm{CAD}}<3=3$, or rank $311: 3^{\mathrm{O}-\mathrm{CAD}}>1=1$ ). Gene changes with a gradient pattern are noted. ID\#, unigene ID number; r1, r2, r3, r4: 8 month-old end-stage Tg53 rats analyzed individually, compared with quiescent-CAD (pooled $n=4)$; and attenuated, quiescent aq-CAD (pooled $n=4$ ); $\times 2$, refers to duplicate spots on each array.

receptors, G-protein $\beta$ subunit, $\mathrm{K}+$ channels, $\alpha 2$ $\mathrm{Na}$, K-ATPase, and genes associated with energy utilization and metabolism (Figs. 2, 4). Assignment to myocardial destabilization is supported by the step-change rank order (Fig. 2).

Although clearly not all inclusive, these paradigms fit in into a putative framework of overt-CAD pathogenesis that is concordant with associated events in acute coronary syndromes: 1) initiating events that involve shear stress and/or increased oxLDL and/or lipid-immune complex entry; 2) plaque destabilization events that involve matrix imbalance, decreased proliferation, inflammation, oxidative stress; and 3) myocardial destabilization involving matrix imbalance, oxidative stress, "pump" dysfunction, metabolic dysfunction (Fig. 2). Within this putative framework, a circuitous interaction is apparent through IL- 1 and IL-18 induced macrophage, neutrophil and T-cell 


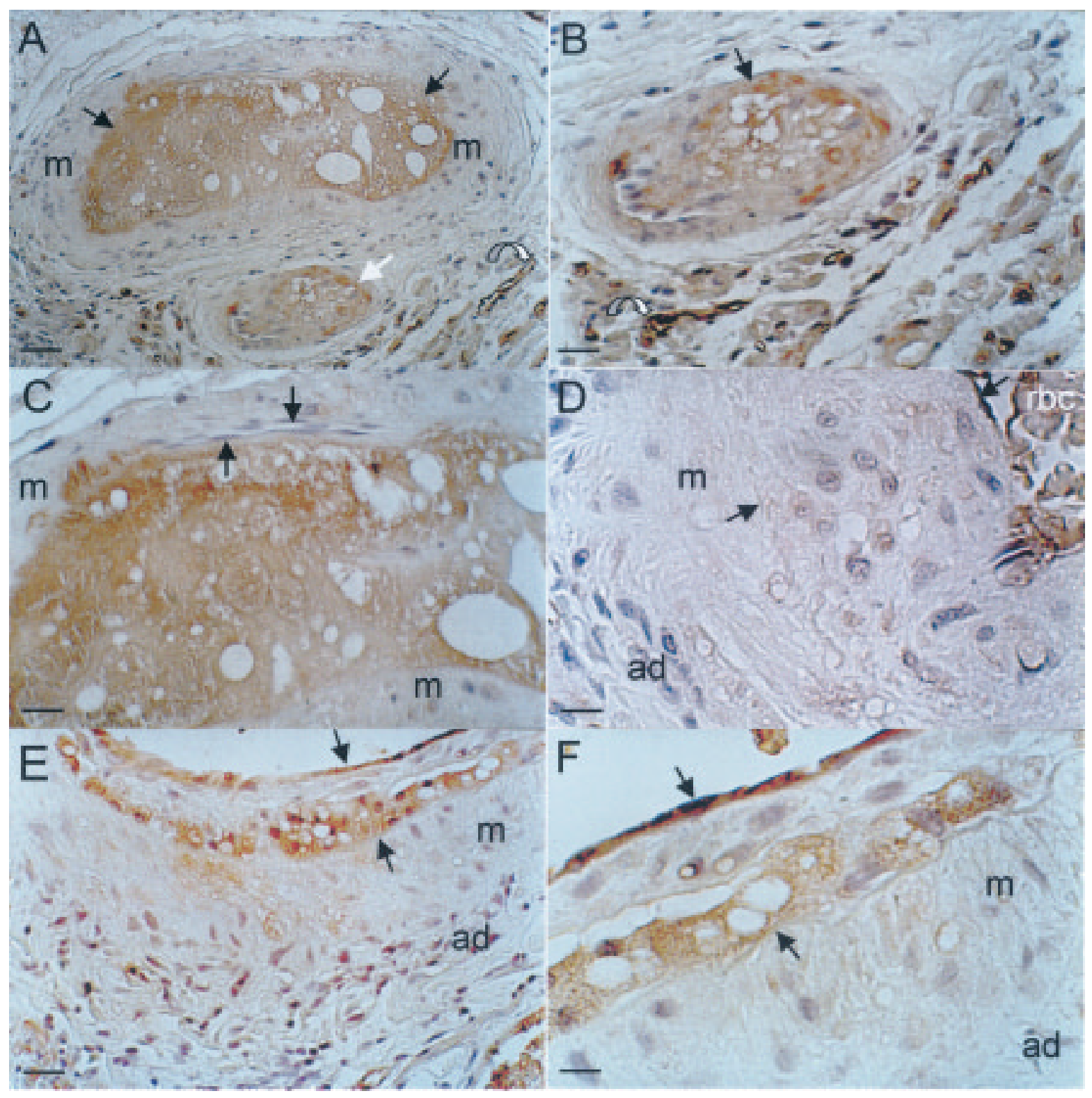

Fig. 3. TIMP-1 immunohistochemical analysis of coronary plaques in end-stage and non-end-stage Tg53 rats. (A), TIMP-1 expression (stained brown) is detected $(\rightarrow$ ) in end-stage Tg53 proximal right coronary "culprit" plaque that is foam-cell rich, with prominent extracellular lipid accumulations, and minimal to no fibrous cap. Bar $=50$ microns. Immunostaining is also detected in endothelial cells of arterioles and capillaries $\left(T_{0}\right)$. Vascular media and adventitia are not stained. (B, C), High magnification (bar $=25$ microns) of plaques depicted in panel (A), corroborating specificity of TIMP-1 immunostaining in culprit plaque foam cells and matrix. Endothelial cells in these lesioned areas $(\rightarrow)$ do not express TIMP-1, in contrast to expression in endothelial cells of intramyocardial arterioles/capillaries ( distal coronary stable plaque $(\rightarrow \leftarrow)$ in end-stage Tg53 RV. Bar $=10$ microns. (E), In attenuated, quiescent-CAD, proximal coronary lesions are smaller and exhibit a cap $(\rightarrow \leftarrow)$. TIMP-1 immunostaining is detected in macrophage foam cells and overlying endothelium. Bar $=25$ microns. (F). High magnification (bar $=10$ microns) of panel E corroborates TIMP-1 immunostaining in plaque macrophage foam cells and endothelium; no immunostaining is detected in the media or adventitia. [Abbreviations: ad, adventitia; m, media; rbc, red blood cells.]

activation-of which activation induces said IL- 1 and IL-18 mediating cytokines. This circuitous interaction could be enhanced by induced lysyl oxidase gene expression, which has been associated with increased monocyte motility (16), as well as by TRHassociated $\mathrm{T}$-cell dependent immune response and increased macrophage superanion-induced oxidative stress. A circuitous interaction suggests the hypothesis of a self-amplifying mechanism consistent with the clinical picture of overt CAD. These observation-based hypotheses remain to be critically tested. 


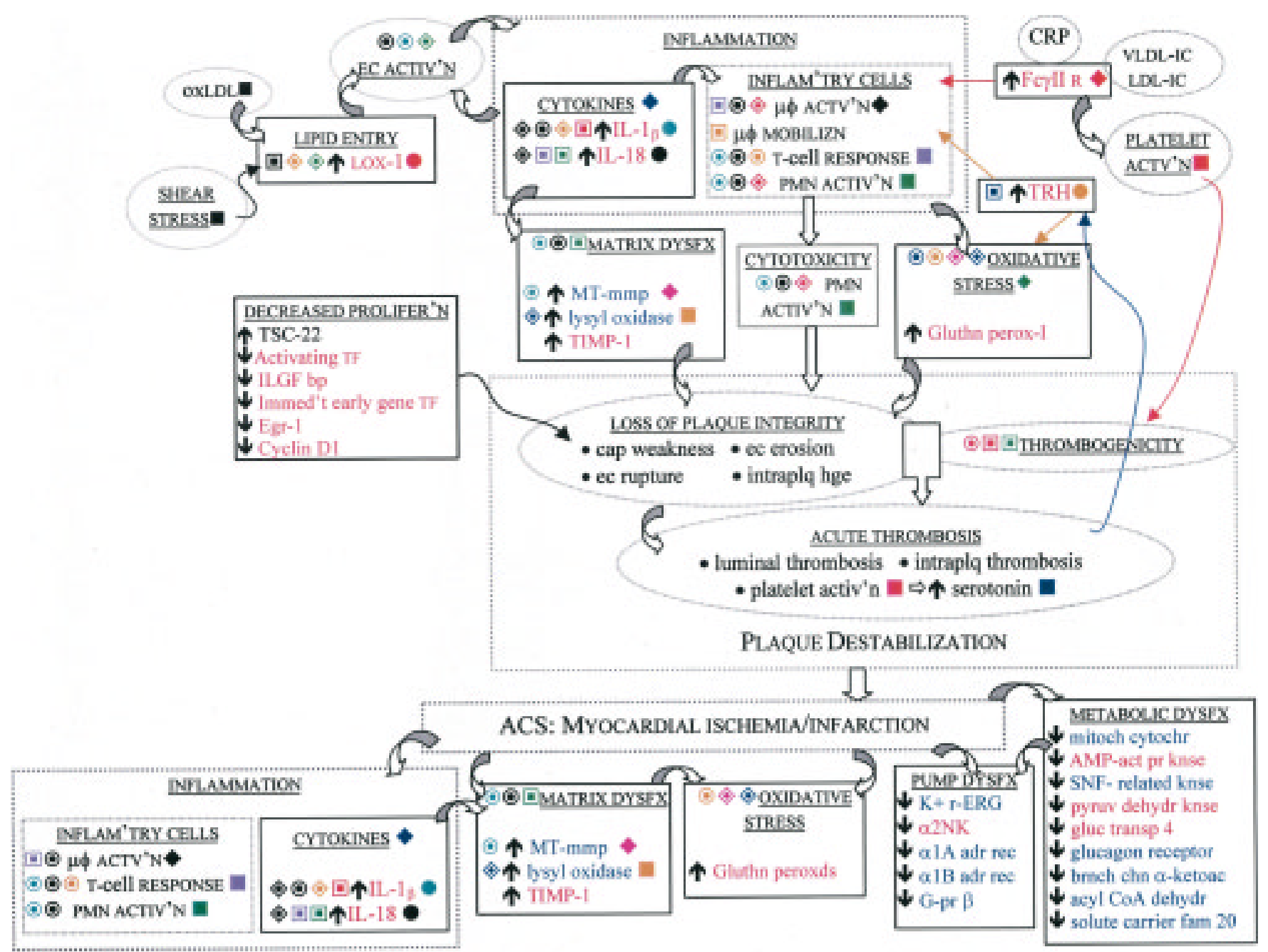

Fig. 4. Putative framework of transcriptional profile changes in overt coronary artery disease. Based on known gene functions, interaction links between turnkey genes exhibiting vectorial changes in overt-CAD compared with both quiescent-CAD controls reveal a circuitous self-propagating interacting gene network that is concordant with emerging pathogenic paradigms of plaque destabilization and post-infarction myocardial injury. Interaction links with known correlates of acute coronary syndromes (shear stress, oxidized LDL, C-reactive protein) are noted. Genes not implicated before in acute coronary syndromes, TRH and Fc $\gamma($ II receptor, also fit in nicely into the proposed self-propagating interacting gene network. Genes printed in red have a gradient rank-order: $\{0-\mathrm{CAD}>$ $\mathrm{q}-\mathrm{CAD}>\mathrm{aq}-\mathrm{CAD}\}$ or $\{\mathrm{o}-\mathrm{CAD}<\mathrm{q}-\mathrm{CAD}<\mathrm{aq}-\mathrm{CAD}\}$; genes printed in blue have a step-change rank order: $\{0-\mathrm{CAD}>\mathrm{q}-\mathrm{CAD}=\mathrm{aq}-$ CAD $\}$ or $\{0-C A D<$ q-CAD $=$ aq-CAD $\}$ (Fig. 2).

Interactions are indicated by like-color and like-symbols: solid symbols induce target (open symbols) matched as to color and shape; $\boldsymbol{\uparrow}$, induction; $\boldsymbol{\Psi}$, deinduction; ACS, acute coronary syndrome; actv'n, activation; acyl CoA dehydrogenase; adrnrg rec, adrenergic receptor; AMP-act pr knse; adenosine-monophosphate-activated protein kinase; branched-chain alpha-keto dehydrogenase subunit El; CRP, C-reactive protein; dysfx, dysfunction; EC, endothelial cell; Egr-1, early growth response-1; Fc $\gamma$ IIr, FC gamma-II receptor; G-pr $\beta$, G-protein $\beta$ subunit; gluthn perox-I, glutathione peroxidase-I; hge, hemorrhage; IL-1 $\beta$, interleukin 1-beta; IL-18, interleukin-18; ILGF-bp3, insulin-like growth factor binding protein-3; intraplq, intraplaque; LDL-IC, low density lipoprotein immune complexes; LOX-1, endothelial oxidized LDL receptor; glucose transporter $4 ; \mu \phi$, macrophage; MT-mmp, membrane-type metalloproteinase; mobilizn, mobilization; occlus'n, occlusion; oxLDL, oxidized LDL, PMN, polymorphonuclear leukocyte or neutrophil; pyruv dehydr knse, pyruvate dehydrogenase kinase; SNF, SNF-related kinase; solute carrier family 20; TSC-22, TGF- $\beta$ stimulated clone 22 transcription repressor; TF, transcription factor; TIMP-1, tissue inhibitor of metalloproteinases-1; TRH, thyrotropin releasing hormone; VEGF-A, vascular endothelial growth factor-A; VLDL-IC, very low density lipoprotein immune complexes.

\section{Discussion}

\section{Differential Functional Gene Cluster Expression Profiles}

The dichotomy of induced and de-induced functional gene clusters is quite compelling and provides confidence in the observations. Transcription profile analysis of aging skeletal muscle also detected a dichotomy of functional gene-cluster induction versus de-induction (17). Functional gene-clusters that are differentially regulated in overt-CAD compared with both quiescent-CAD controls are most likely to be involved in overt-CAD pathogenesis, and that specific genes that are induced or deinduced in said clusters, most likely play putative turnkey roles in overt- 
CAD. These turnkey genes implicate upregulation of inflammation, matrix changes, oxidative stress response to a presumed oxidative stress, oxidized LDL entry, and a downregulation of proliferation, angiogenesis, adrenergic receptor system, metabolism, and ion channels and transporters-which altogether assign a complex molecular framework underlying overt-CAD that is essentially concordant with known pathophysiological dysfunction in human acute coronary syndrome.

Fully cognizant that specific gene-based pathway determination is limited by the 1046-gene array, deduced framework concepts can assume more definitiveness. The data, whether through a 1000 or 10,000 array, demonstrates a framework concept that transcriptional mechanisms are involved, and suggests the hypothesis that quiescentto-overt CAD is an active transcriptionally mediated pathogenic process and not just a secondary "wearand-tear" disruption-thrombosis event.

\section{Putative Threshold-players}

The identification of reproducible differential gene regulation in overt-CAD in end-stage Tg53 rat hearts compared with age-matched non-overt CAD (nonendstage Tg53-reg) and attenuated non-overt CAD (non-endstage Tg53-lsd) rat hearts supports the concept of putative threshold-gene players. At this stage, since the array is limited to 1046 known genes, the full complement of threshold-gene players is not possible, however, a putative first list of threshold-players is drawn (Fig. 2), which provides concordance with previous histological and immunohistochemical analyses (8), as well as novel insight deserving mention. For example, TIMP-1 induction aligns with plaque matrix stabilization and its observed robust induction would be counterintuitive to plaque destabilization $(18,19)$ or myocardial destabilization. However, TIMP-1 has been identified as a robust marker for invasive cancer rather than the expected metalloproteinases (14), and has also been detected to be upregulated in premature coronary atherosclerosis (20). This suggests that TIMP-1 might also be a compensatory response serving as pragmatic biomarker for overt-CAD or acute coronary syndromes, or that other putative TIMP-1 functions remain to be elucidated, reminiscent of the "other non-matrix related functions" of metalloproteinases (21). Likewise, lysyl oxidase is associated with stabilization of collagen fibers by crosslinking (22). However, new data suggest that lysyl oxidase is associated with enhanced monocyte motility (15) suggesting the hypothesis that the observed induction in overt-CAD could facilitate macrophage-mediated plaque and/or myocardial destabilization pathways. The robust 25- to 29-fold induction of TRH in ventricular samples is deduced to be specific for vascular adventitial and endothelial expression, and not ventricular cardiomyocytes based on reported in situ hybridization observations (23).
Because TRH has been shown to play a critical role in T-cell mediated immune response (24) and enhances superanion production of activated macrophages (25), we hypothesize that induced adventitial TRH represents a paracrine vascular pathway that could be expected to contribute to inflammatory cascades in plaque destabilization, as well as contribute to myocardial injury response. The induction of Fc $\gamma$ II receptor suggests a possible role for lipoprotein-immune complex uptake and Fc $\gamma$ II receptor activation-induced inflammatory mechanisms in overt CAD. This notion is supported by the detection of Fc $\gamma$ II receptors in human atherosclerotic lesions (26). Additionally, we note that C-reactive protein, increasingly implicated as a coronary risk marker (27), binds to Fc $\gamma$ II receptors on monocytes and neutrophils (28). The induction of both IL-1 and IL-18 supports the hypothesis of a self-propagating upregulation of inflammatory response mediated by neutrophils, macrophages and/or T-cells (29-32), which most likely contributes to plaque destabilization, as well as postischemia/infarction myocardial destabilization. These hypotheses are supported by the detection of IL-1 and IL-18 in human atherosclerotic plaques and relation to plaque instability (33), and implication in human myocardial ischemic dysfunction (34).

\section{Transcriptional Framework of Overt-CAD}

Having controlled genetic background, age, gender, diet, and environment, the transcriptional profile of overt-CAD exhibits detectable distinct functional gene cluster regulation compared with quiescent-CAD. Although limited to a 1046-gene array, the robust and reproducible transcription profile results associated with overt-CAD in end-stage $\mathrm{Tg} 53$ rats, and its distinction from transcription profiles detected in mouse LAD-ligated myocardial infarction model (7) and in a shear-stress vascular smooth muscle cell model (35), strongly support the specificity of our observations for atherosclerotic overt-CAD.

Altogether, the data reveal that transition to overtCAD involves a transcriptionally-active pathogenic framework with a set of turnkey genes which, based on known function and interaction, comprise a selfamplifying interrelated gene-network, that can drive processes linked to both coronary plaque destabilization and post-event myocardial destabilization such as matrix degradation, inflammation, lipidentry, and de-induced proliferation and angiogenesis, in the presence of an altered state of metabolism, ion transporters and alpha-adrenergic receptor system. Although full investigation of all turnkey genes remains to be done, the experimental delineation of, and integrated insight into the putative multi-gene cluster transcriptional framework of overt-CAD is a key first step. The identification of said transcriptional molecular framework suggests the hypothesis that overt-CAD involves altered "hardwiring" which, a priori, would require multi-faceted targeted 
intervention directed at both plaque and myocardial destabilization.

\section{Acknowledgments}

We acknowledge excellent technical assistance of Kimberly Zander and Sarah T. Cruz. This work was supported in part by a grant from the NIH National Heart, Lung, and Blood Institute (HL 62857) and by a grant-in-aid from the American Heart Association.

\section{References}

1. Libby P, Geng YJ, Sukhova GK, et al. (1997) Molecular determinants of atherosclerotic plaque vulnerability. Ann. N.Y. Acad. Sci. 811: 134-142.

2. Zhou J, Chew M, Ravn HB, Falk E. (1999) Plaque pathology and coronary thrombosis in the pathogenesis of acute coronary syndromes. Scan. J. Clin. Lab. Invest. 230: 3-11.

3. Delager-Pedersen S, Pederson EM, Ringgaard S, Falk E. (1999) In: Fuster V, (ed.) The Vulnerable Atherosclerotic Plaque. Futura, NY, pp. 1-23.

4. Frangogiannis NG, Smith CW, Entman ML. (2002) The inflammatory response in myocardial infarction. Cardiovasc. Res. 53: 31-47.

5. Virmani R, Kolodgie FD, Burke AP, et al. (2000) Lessons from sudden coronary death: a comprehensive morphological classification scheme for atherosclerotic lesions. Arterioscler. Thromb. Vasc. Biol. 20: 1262-1275.

6. Glagov S, Rowley DA, Kohut RI. (1961) Atherosclerosis of human aorta and its coronary and renal arteries. Arch. Pathol. 72: 82-95.

7. Herrera VLM, Makarides SC, Xie HX, et al. (1999) Spontaneous combined hyperlipidemia, coronary heart disease and decreased survival in Dahl salt-sensitive hypertensive rats transgenic for human cholesteryl ester transfer protein. Nat. Med. 5: 1383-1389.

8. Herrera VLM, Didishvili T, Lopez LV, et al. (2001) Hypertension exacerbates coronary artery disease in transgenic hyperlipidemic Dahl salt-sensitive hypertensive rats. Mol. Med. 7: 831-844.

9. Lyn D, Liu X, Bennett NA, Emmett NL. (2000) Gene expression profile in mouse myocardium after ischemia. Physiol. Genomics 2: 93-100.

10. Gilles C, Polette M, Piette J, et al. (1996) High level of MTMMP expression is associated with invasiveness of cervical cancer cells. Int. J. Cancer 65: 209-213.

11. Jin L, Yuan RQ, Fuchs A, et al. (1997) Expression of interleukin-1beta in human breast carcinoma. Cancer 80: 42 1-434.

12. Apte RN, Dvorkin T, Song X, et al. (2000) Opposing effects of IL- 1 alpha and IL- 1 beta on malignancy patterns. Tumor cellassociated IL-1 alpha potentiates anti-tumor immune responses and tumor regression, whereas IL-1 beta potentiates invasiveness. Adv. Exp. Med. Biol. 479: 277-288.

13. Belkin AM, Akimov SS, Zaritskaya LS. (2001) Matrixdependent proteolysis of surface transglutaminase by membrane-type metalloproteinase regulates cancer cell adhesion and locomotion. J. Biol. Chem. 276: 18415-18422.

14. Yoshikawa T, Tsuburaya A, Kobayashi O, et al. (2001) Intratumoral concentrations of tissue inhibitor of matrix metalloproteinase 1 in patients with gastric carcinoma: a new biomarker for invasion and its impact on survival. Cancer 91: 1739-1744.
15. Kirschman DA, Seftor EA, Nieva DR, et al. (1999) Differentially expressed genes associated with the metastatic phenotype in breast cancer. Breast Cancer Res. Treat. 55: 127-136.

16. Lazarus HM, Cruikshank WW, Narashimhan N, et al. (1995) Induction of human monocyte motility by lysyl oxidase. Matrix Biol 14: 727-731.

17. Lee CK, Klopp RG, Weindruch R, Prolla TA. (1999) Gene expression profile of aging and its retardation by caloric restriction. Science 285: 1390-1394.

18. Fabunmi RP, Sukhova GK, Sugiyama S, Libby P. (1998) Expression of tissue inhibitor of metalloproteinases- 3 in human atheroma and regulation in lesion-associated cells: a potential protective mechanism in plaque stability. Circ. Res. 83: 270-278.

19. Rouis M, Adamy C, Duverger N, et al. (1999) Adenovirusmediated overexpression of tissue inhibitor of metalloproteinase-1 reduces atherosclerotic lesions in apolipoprotein E-deficient mice. Circulation 100: 533-540.

20. Noji Y, Kajinami K, Kawashiri MA, et al. (2001) Circulating matrix metalloproteinases and their inhibitors in premature coronary atherosclerosis. Clin. Chem. Lab. Med. 39: 380-384.

21. Coussens LM, Fingleton B, Matrisian LM. (2002). Matrix metalloproteinases inhibitors and cancer: trials and tribulations. Science 295: 2387-2392.

22. Csiszar K. (2001) Lysyl oxidases: a novel multifunctional amine oxidase family. Prog. Nucleic Acid Res. Mol. Biol. 70: 1-32.

23. Shi ZX, Xu W, Mergner WJ, et al. (1996) Localization of thyrotropin-releasing hormone mRNA expression in the rat heart by in situ hybridization histochemistry. Pathobiology 64: 314-319.

24. Perez Castro C, Penalva R, Paez Pereda M, et al. (1999) Early activation of thyrotropin-releasing-hormone and prolactin plays a critical role during a $\mathrm{T}$ cell-dependent immune response. Endocrinology 140: 690-697.

25. Koshida H, Kotake Y. (1993) Thyrotropin-releasing hormone enhances the superoxide anion production of rabbit peritoneal macrophages stimulated with $\mathrm{N}$-formyl-methionyl-leucylphenylalanine and opsonized zymosan. Life Sci. 53: 725-731.

26. Ratcliffe NR, Kennedy SM, Morganelli PM. (2001) Immunocytochemical detection of Fc-gamma receptors in human atherosclerotic lesions. Immunol. Let. 77: 169-174.

27. Zebrack JS, Anderson JL, Maycock CA, et al. (2002) Usefulness of high-sensitivity $\mathrm{C}$-reactive protein in predicting longterm risk of death or acute myocardial infarction in patients with unstable or stable angina pectoris or acute myocardial infarction. Am. J. Cardiol. 89: 145-149.

28. Chi M, Tridandapani S, Zhong W, et al. (2002) C-reactive protein induces signaling through Fc-gamma RIIa on HL-60 granulocytes. J. Immunol. 168: 1413-1418.

29. Dinarello CA. (1999) Interleukin-18. Methods 19: 121-132.

30. Grutkoski PS, D'Amico R, Ayala A, Simms HH. (1999) Il-1 beta stimulation induces paracrine regulation of PMN function and apoptosis. Shock 12: 373-381.

31. Leung BP, Culshaw S, Gracie JA, et al. (2001) A role for IL18 in neutrophil activation. J. Immunol. 167: 2879-2886.

32. Ogura T, Ueda H, Hosohara K, et al. (2001) Interleukin-18 stimulates hematopoietic cytokine and growth factor formation and augments circulating granulocytes in mice. Blood 98: 2101-2107.

33. Mallat Z, Corbaz A, Scoazec A, et al. (2001) Expression of interleukin-18 in human atherosclerotic plaques and relation to plaque instability. Circulation 104: 1598-1603.

34. Pomerantz BJ, Reznikov LL, Harken AH, Dinarello CA. (2002) Inhibition of caspase 1 reduces human myocardial ischemic dysfunction via inhibition of IL-18 and IL-1 $\beta$. Proc. Natl. Acad. Sci. 98: 2871-2876.

35. Feng Y, Yang JH, Huang H, et al. (1999) Transcriptional profile of mechanically induced genes in human vascular smooth muscle cells. Circ. Res. 85: 1118-1123. 